\title{
Cytokine and Chemokine Profile Changes in Patients After Intravitreal Conbercept Injection for Diabetic Macular Edema
}

This article was published in the following Dove Press journal:

Drug Design, Development and Therapy

\author{
Qingquan Wei ${ }^{1, *}$ \\ Zhongqi Wan ${ }^{2, *}$ \\ Yongcheng $\mathrm{Hu}^{3, *}$ \\ Qing Peng'
}

'Department of Ophthalmology, Shanghai Tenth People's Hospital, Tongji University, Shanghai 200072, People's Republic of China; ${ }^{2}$ School of Public Health, Nanjing Medical University, Nanjing 2III66, Jiangsu Province, People's Republic of China; ${ }^{3}$ Department of Ophthalmology, Bayannuer Paralympic Eye Hospital, Inner Mongolia 0I5000, People's Republic of China

*These authors contributed equally to this work
Correspondence: Qing Peng

Department of Ophthalmology, Shanghai

Tenth People's Hospital, Tongji University,

Shanghai 200072, People's Republic of

China

Tel +86 I32208II 345

Email pengqing@tongji.edu.cn
Purpose: This study aimed to investigate the concentrations of cytokines and chemokines in diabetic macular edema (DME) eyes before and during therapy with the intravitreal injection of conbercept (IVC) and to identify associations with disease activity.

Methods: The Bio-Plex ${ }^{\circledR} 200$ System and the Bio-PlexTM Human Cytokine Standard 27-Plex, Group I (Bio-Rad, Hercules, California, USA) were used to detect cytokine levels in aqueous humour. Experimental aqueous humour samples were collected from 18 patients with DME at the same time that IVC was performed at baseline and at 1 month. Control aqueous humour samples were collected from 16 patients undergoing cataract surgery.

Results: Significantly higher concentrations of vascular endothelial growth factor (VEGF), interleukin 6 (IL-6), IL-8, eotaxin, granulocyte colony stimulating factor (G-CSF), interferon gamma-induced protein 10 (IP-10), and monocyte chemoattractant protein-1 (MCP-1) were found in the aqueous humour of DME patients than cataract patients. One month after IVC, the intraocular concentrations of VEGF were significantly lower in the eyes of DME patients than at baseline. No other cytokines were significantly altered by conbercept therapy. Bestcorrected visual acuity (BCVA) slightly improved following IVC compared with that at baseline, although this difference was not significant, and central macular thickness (CMT) significantly decreased 1 month after IVC treatment.

Conclusion: Angiogenic, inflammatory and growth factors are involved in the development of DME. With the exception of VEGF, IVC did not cause significant differences in any inflammatory cytokines or growth factors in DME patients. CMT is related to VEGF levels in aqueous humour.

Keywords: diabetic macular oedema, conbercept, intravitreal implant, intraocular cytokines

\section{Introduction}

Diabetic macular edema (DME) is a major cause of visual impairment in diabetic patients with diabetic retinopathy (DR) in economically developed societies. ${ }^{1,2}$ Vascular endothelial growth factor (VEGF) is an important angiogenic factor and plays an important role in the pathogenesis of DME; therefore, anti-VEGF treatments for DME have been developed. ${ }^{3,4}$ Currently, there are many anti-VEGF drugs, such as bevacizumab, ranibizumab, and aflibercept, that show some slight differences in terms of their ability to block VEGF isoforms; the efficacy of these drugs is similar in DME patients, causing relative vision improvements. ${ }^{5,6}$ Conbercept is a fusion protein comprising extracellular domain-2 of vascular endothelial growth factor receptor (VEGFR)-1 and extracellular domain-3 and 4 of VEGFR-2. ${ }^{7}$ Conbercept 
has a superior binding affinity to VEGF than ranibizumab. ${ }^{8}$ Studies have shown that anti-VEGF treatment effectively improved visual acuity (VA) and reduced macular thickness in DME patients. ${ }^{9}$ Unfortunately, some patients showed no significant improvement or experienced the recurrence of DME despite monthly intravitreal anti-VEGF therapy. ${ }^{10}$ Therefore, mechanisms other than VEGF may contribute to DME in DR.

There has been an increasing amount of evidence showing that inflammation contributes to the pathogenesis of DR. ${ }^{11,12}$ DME formation is driven by the breakdown of the bloodretina barrier and the consequent vascular leakage and thickening of the retina, thus causing thickening, macular malfunction, and visual impairment. ${ }^{13}$ Cellular events such as the increased expression of inflammatory mediators, leucostasis, and abnormal leukocyte adherence result in increased vascular permeability in the pathogenesis of DME. ${ }^{14,15}$ It has been reported that the levels of VEGF, interleukin (IL)-6, IL-8, intercellular adhesion molecule-1 (ICAM-1), interferon gamma-induced protein-10 (IP-10), and monocyte chemoattractant protein-1 (MCP-1) are increased in DME patients compared with diabetic patients without DME. ${ }^{16}$ However, the exact mechanisms and reasons for the response to antiVEGF treatment in DME patients remain unclear. Accordingly, this study investigated the cytokine profiles in the aqueous humour of patients with DME and investigated the effect of therapy with the intravitreal injection of conbercept (IVC) on angiogenic and inflammatory cytokines.

\section{Materials and Methods Subjects}

This study was conducted at the Department of Ophthalmology, Shanghai Tenth People's Hospital affiliated with Tongji University School of Medicine. This study was approved by the Research Ethics Committee, Shanghai Tenth People's Hospital affiliated with Tongji University School of Medicine, Shanghai, China. The procedures conformed to the tenets of the Declaration of Helsinki. Written informed consent was obtained from each participant for all examinations and procedures.

We included patients who were treated with IVC from September 2017 to January 2019. All patients received an intravitreal injection of $0.05 \mathrm{~mL}(0.5 \mathrm{mg})$ conbercept (Chengdu Kanghong Biotechnologies Co., Ltd.; Chengdu, Sichuang, China). Reference samples were obtained from patients undergoing cataract surgery. The inclusion criteria for DME were as follows: (1) diffuse ME based on fundus fluorescein angiography (FFA), (2) a decrease in VA, (3) CMT greater than $250 \mu \mathrm{m}$ based on optical coherence tomography (OCT) and (4) a follow-up visit at least 1 month after IVC. The exclusion criteria were as follows: (1) previous ocular surgery; (2) glaucoma, vitreous haemorrhaging or tractional retinal detachment (TRD) before IVC; (3) prior injections with medications; and (4) macular edema (ME) caused by any retinal condition other than DR.

\section{Fundus Findings}

Ophthalmic examinations were performed before and after IVC. Examinations included a best-corrected visual acuity (BCVA) test using manifest refraction, the Logarithm of the Minimum Angle of Resolution (logMAR) VA chart, slit-lamp examinations, fundus examinations and FFA, which was assessed using a fundus camera (TRC-50EX; Tokyo Optical, Tokyo, Japan). CMT was calculated as the distance from the inner limiting membrane to the basal membrane of the retinal pigment epithelium. CMT was defined as the value of a 1-mm central area based on OCT (Zeiss-Humphrey, Dublin, California, USA).

\section{Sample Collection}

All patients with DR received intravitreal injections of $0.05 \mathrm{~mL}$ $(0.5 \mathrm{mg})$ conbercept. Aqueous humour samples were taken at the same time that IVC was performed at baseline and at 1 month. A mean volume of $200 \mu \mathrm{L}$ aqueous humour was collected via anterior chamber paracentesis. Immediately after collection, aqueous humour samples were transferred to sterile plastic tubes and were stored at minus $80^{\circ} \mathrm{C}$ until analysis. Control aqueous samples were collected from sixteen patients undergoing routine cataract surgery by limbal paracentesis, and the samples were frozen and stored at minus $80^{\circ} \mathrm{C}$.

\section{Measurement of Cytokines}

The Bio-Plex ${ }^{\circledR} 200$ System and Bio-Plex ${ }^{\mathrm{TM}}$ Human Cytokine Standard 27-Plex, Group I (Bio-Rad, Hercules, California, USA) were used according to the manufacturer's instructions to analyse aqueous humour samples. The selection of cytokines for detection was based on previous studies. ${ }^{16-19}$ The following cytokines were analysed: platelet-derived growth factor bb (PDGFF-bb), interleukin-1 beta (IL-1 $\beta$ ), interleukin-1 receptor antagonist (IL-1 ra), IL-2, IL-4, IL-5, IL-6, IL-7, IL-8, IL-9, IL-10, IL-12 (p70), IL-13, IL-15 and IL-17, eotaxin, basic fibroblast growth factor (FGF basic), granulocyte colony stimulating factor (G-CSF), granulocytemacrophage colony-stimulating factor (GM-CSF), interferon 
gamma (IFN)- $\gamma$; IP-10, MCP-1, macrophage inflammatory protein 1 alpha and 1 beta (MIP-1 $\alpha$ and MIP-1 $\beta$ ), regulated on activation $\mathrm{T}$ cell expressed and secreted (RANTES); tumour necrosis factor alpha (TNF- $\alpha$ ), and VEGF.

\section{Statistical Analysis}

SPSS V.19.0 for Windows (SPSS, Chicago, Illinois, USA) was used to analyse the data. A one-sample KolmogorovSmirnov test was used to examine whether the samples were normally distributed. A Student's $t$-test was employed to compare normally distributed, unpaired, continuous variables between the two groups. The Mann-Whitney $U$-test was used for variables with a skewed distribution. The $\chi^{2}$ test or Fisher's exact test was performed to compare discrete variables. Spearman's rank-order correlation coefficients or Pearson's correlation coefficients were calculated to examine the relationships among the variables. $\mathrm{P}<0.05$ was defined as statistically significant.

\section{Results}

\section{Baseline Characteristics and Patient Demographics}

This study consisted of 34 patients, which included 18 patients in the DME group and 16 non-retinal disease patients with cataracts (the control group). As shown in Table 1, the general clinical characteristics, such as age, sex and the proportion of patients with hypertension, were not significantly different between the DME group and the control group $(\mathrm{p}>0.05)$. The mean duration of DME symptoms was 12.48 months (Table 1).

\section{Influence of Conbercept Treatment on Clinical Parameters}

As shown in Table 2, BCVA slightly improved following IVC compared with baseline, although this difference was not significant $(\mathrm{p}=0.10)$. In patients with DME, the mean

Table I Demographic Characteristics of the Patients in the DME and Control Groups

\begin{tabular}{|l|l|l|l|}
\hline & $\begin{array}{l}\text { DME } \\
(\mathbf{n}=18)\end{array}$ & $\begin{array}{l}\text { Control } \\
(\mathbf{n}=16)\end{array}$ & p value \\
\hline Age, years & $57.72 \pm 6.81$ & $63.94 \pm 3.85$ & 0.18 \\
Gender (female), \% & 44.44 & 43.81 & 0.97 \\
With hypertension, \% & 33.33 & 25 & 0.72 \\
Duration of symptoms, months & $12.48 \pm 4.03$ & NA & NA \\
\hline
\end{tabular}

Notes: The data are presented as the mean $\pm S D$, number (\%) or median (range). Abbreviations: DME, diabetic macular edema; NA, not applicable. baseline CMT was $609.06 \mu \mathrm{m}$, and this value decreased to $412.50 \mu \mathrm{m} 1$ month after treatment $(\mathrm{p}=0.0047)$. In the DME group, no patients in the control or IVC groups experienced vitreous haemorrhage, rubeosis iridis, fibroneovascular membranes or TRD.

\section{Concentrations of Cytokines at Baseline}

The measurement of aqueous humour cytokine levels showed that there were significantly higher concentrations of IL-6, IL-8, eotaxin, G-CSF, IP-10, MCP-1, and VEGF in the DME group than in the control group. The levels of IL-1ra, IL-7, IL-7, IL-9, eotaxin, G-CSF, MIP- $1 \alpha$, and MIP-1 $\beta$ were not different between the DME group and the control group. The concentrations of the other cytokines were outside of the range of the minimum detectable concentrations (Figure 1).

We also assessed the significance of relationships among the cytokine levels in aqueous humour before IVC in DME. There were significant correlations between the level of IL-6 and the levels of IL-8, eotaxin, G-CSF, and MCP-1. There were also significant correlations between the level of IL-8 and the levels of eotaxin and MCP-1. Furthermore, there were significant correlations between the level of eotaxin and the levels of IP-10 and MCP-1. However, there was no significant correlation between the levels of VEGF and those of IL-6, IL-8, eotaxin, G-CSF, IP-10, and MCP-1 (Table 3).

\section{Changes in Cytokine Levels During Treatment}

The intraocular concentrations of VEGF significantly decreased in DME eyes one month after the first IVC treatment compared with those at baseline $(p<0.0001)$. All other cytokines were unaffected by conbercept therapy,

Table 2 Clinical Characteristics of the Patients in the DME Group

\begin{tabular}{|l|l|l|l|}
\hline & $\begin{array}{l}\text { DME Before } \\
\text { IVC }\end{array}$ & $\begin{array}{l}\text { DME After } \\
\text { IVC }\end{array}$ & p value \\
\hline BCVA (logMAR) & $0.89 \pm 0.31$ & $0.71 \pm 0.31$ & 0.10 \\
CMT $(\mu \mathrm{m})$ & $609.06 \pm 218.47$ & $4 \mid 2.50 \pm 168.26$ & $0.0047^{*}$ \\
\hline Vitreoretinal condition, no. & & & \\
Vitreous haemorrhage & 0 & 0 & - \\
Rubeosis iridis & 0 & 0 & - \\
Fibroneovascular & 0 & 0 & - \\
membranes & & 0 & - \\
TRD & 0 & 0 & - \\
\hline
\end{tabular}

Notes: The data are presented as the mean \pm SD or numbers. *Indicates $p<0.05$. Abbreviations: BCVA, best corrected visual acuity; CMT, central macular thickness; DME, diabetic macular edema; IVC, intravitreal injection of conbercept; logMAR, logarithm of the minimum angle of resolution; TRD, tractional retinal detachment. 

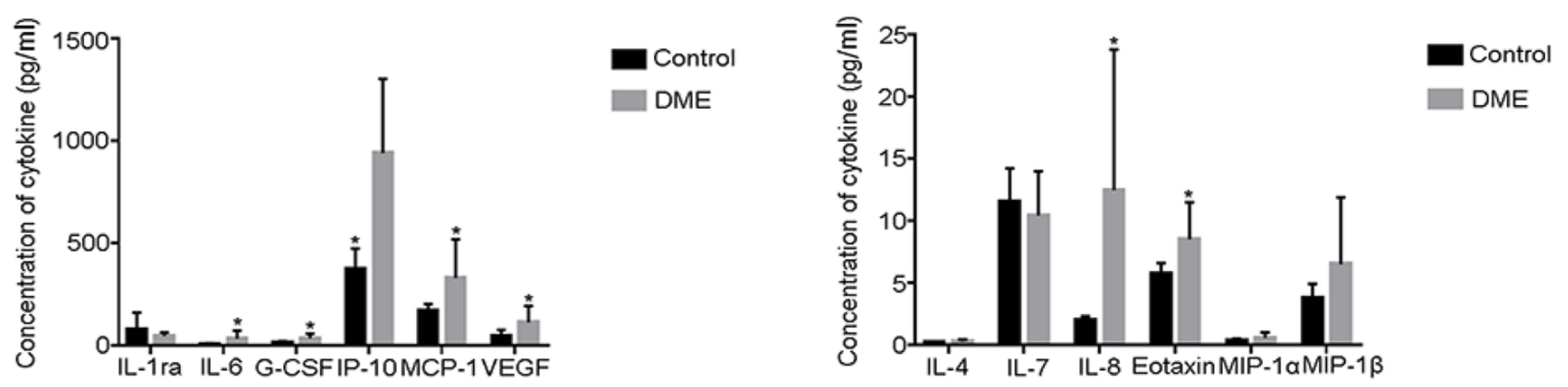

Figure I Clinical characteristics of the patients in the DME group. *Indicates DME vs control: $p<0.05$.

but some cytokine levels were outside of the range of the minimum detectable concentrations (Figure 2).

The decrease in VEGF levels was associated with a decrease in CMT and an improvement in VA one month after the first IVC treatment. There were significant correlations between the level of VEGF and CMT $(r=0.504, p=0.033$ ) (Figure 3A). There were no significant correlations between the level of VEGF and BCVA ( $\mathrm{r}=0.455, \mathrm{p}=0.058$ ) (Figure 3B). There were also no significant correlations between the level of CMT and BCVA ( $\mathrm{r}=0.256, \mathrm{p}=0.304)$ (Figure 1C). A representative case of the response of a patient with DME to IVC therapy, as documented by fundus photography, OCT, BCVA, and VEGF concentrations, is shown (Figure 4).

\section{Discussion}

In the present study, we demonstrated that the aqueous humour levels of IL-6, IL-8, eotaxin, G-CSF, IP-10, MCP1 , and VEGF were significantly higher in eyes with DME than in control eyes with cataracts. IL-6 is a multifunctional cytokine that plays an important role in the induction of acute inflammatory responses and the regulation of immune processes..$^{20}$ IL-8 also plays a pivotal role in the regulation of the acute inflammatory response to invading pathogens. ${ }^{21}$ Eotaxins belong to a family of chemokines, and these small secreted peptides are important immune modulators that act as potent chemoattractants for eosinophils and other types of cells. ${ }^{22} \mathrm{G}-\mathrm{CSF}$ is a haemopoietic growth factor that promotes the survival, proliferation, differentiation, and function of granulocytes. G-CSF also increases the number of circulating

Table 3 Correlations Between Aqueous Humour Factors Before IVC in DME

\begin{tabular}{|c|c|c|c|c|c|c|c|}
\hline \multirow[t]{3}{*}{ Variable } & IL-6 & IL-8 & Eotaxin & G-CSF & IP-IO & MCP-I & VEGF \\
\hline & $\mathbf{r}$ & $r$ & $r$ & $r$ & $r$ & $r$ & $r$ \\
\hline & p value & p value & $P$ value & p value & p value & p value & p value \\
\hline IL-6 & & $\begin{array}{l}0.618 \\
0.019 *\end{array}$ & $\begin{array}{l}0.644 \\
0.013^{*}\end{array}$ & $\begin{array}{l}0.739 \\
0.003^{*}\end{array}$ & $\begin{array}{l}0.459 \\
0.098\end{array}$ & $\begin{array}{l}0.855 \\
<0.00 I^{*}\end{array}$ & $\begin{array}{l}0.544 \\
0.130\end{array}$ \\
\hline IL-8 & & & $\begin{array}{l}0.600 \\
0.023^{*}\end{array}$ & $\begin{array}{l}0.139 \\
0.637\end{array}$ & $\begin{array}{l}0.130 \\
0.659\end{array}$ & $\begin{array}{l}0.705 \\
0.005^{*}\end{array}$ & $\begin{array}{l}0.151 \\
0.699\end{array}$ \\
\hline Eotaxin & & & & $\begin{array}{l}0.411 \\
0.144\end{array}$ & $\begin{array}{l}0.587 \\
0.027^{*}\end{array}$ & $\begin{array}{l}0.609 \\
0.02 I^{*}\end{array}$ & $\begin{array}{l}0.460 \\
0.213\end{array}$ \\
\hline G-CSF & & & & & $\begin{array}{l}0.480 \\
0.083\end{array}$ & $\begin{array}{l}0.532 \\
0.050\end{array}$ & $\begin{array}{l}0.510 \\
0.160\end{array}$ \\
\hline IP-10 & & & & & & $\begin{array}{l}0.405 \\
0.154\end{array}$ & $\begin{array}{l}0.126 \\
0.748\end{array}$ \\
\hline MCP-I & & & & & & & $\begin{array}{l}0.159 \\
0.683\end{array}$ \\
\hline
\end{tabular}

Note: *Indicates $\mathrm{p}<0.05$.

Abbreviations: IL, interleukin; IP- I0, interferon gamma-induced protein I0; MCP-I monocyte chemoattractant protein-I; VEGF, vascular endothelial growth factor. 

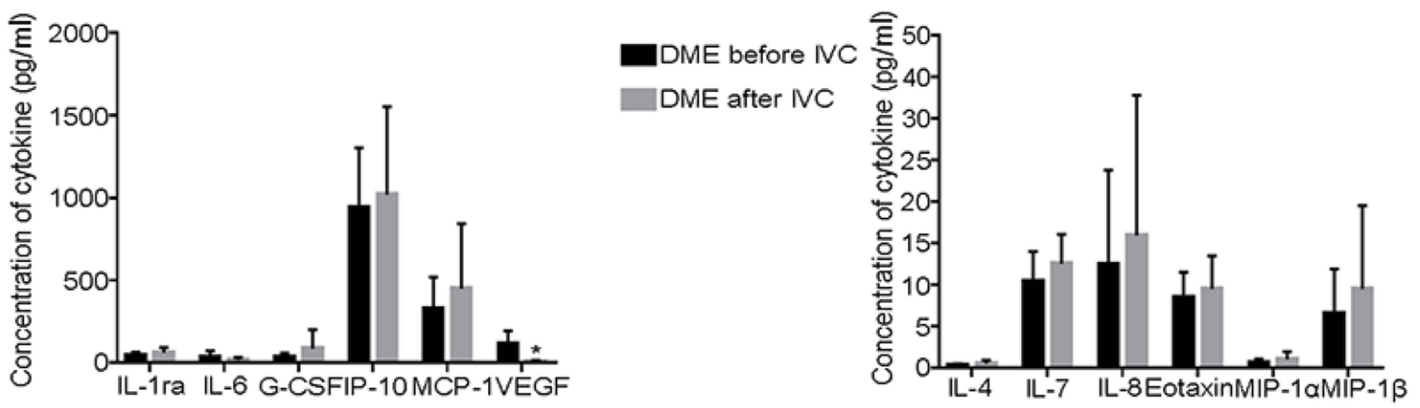

DME before IVC

DME after IVC

Figure 2 Baseline concentrations of the cytokines and chemokines. *Indicates DME after IVC vs DME before IVC: $p<0.05$
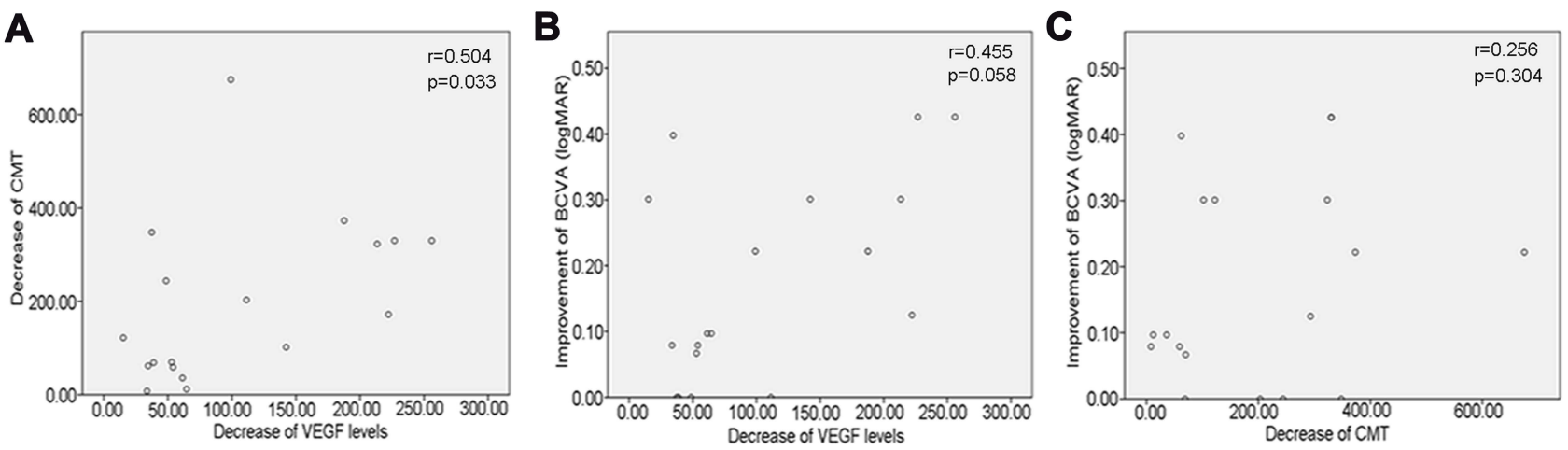

Figure 3 Correlation among the change in VEGF, best-corrected visual acuity (BCVA) and central macular thickness (CMT) I month after intravitreal injection of conbercept (IVC). (A) Significant positive correlation between the decrease in VEGF and the decrease in CMT I month after IVC. (B) No significant correlation between the decrease in VEGF and the improvement in BCVA I month after IVC. (C) No significant correlation between the improvement in BCVA and the decrease in CMT I month after IVC.

leukocytes and changes the human immune system. ${ }^{23} \mathrm{IP}-10$ is secreted by several cell types, such as monocytes, endothelial cells, and fibroblasts. IP-10 is a potent chemoattractant that induces the migration of monocytes and activates T-cells during inflammation. ${ }^{24,25} \mathrm{MCP}-1$, also referred to as chemokine (C-C motif) ligand 2 (CCL2), plays a critical role in
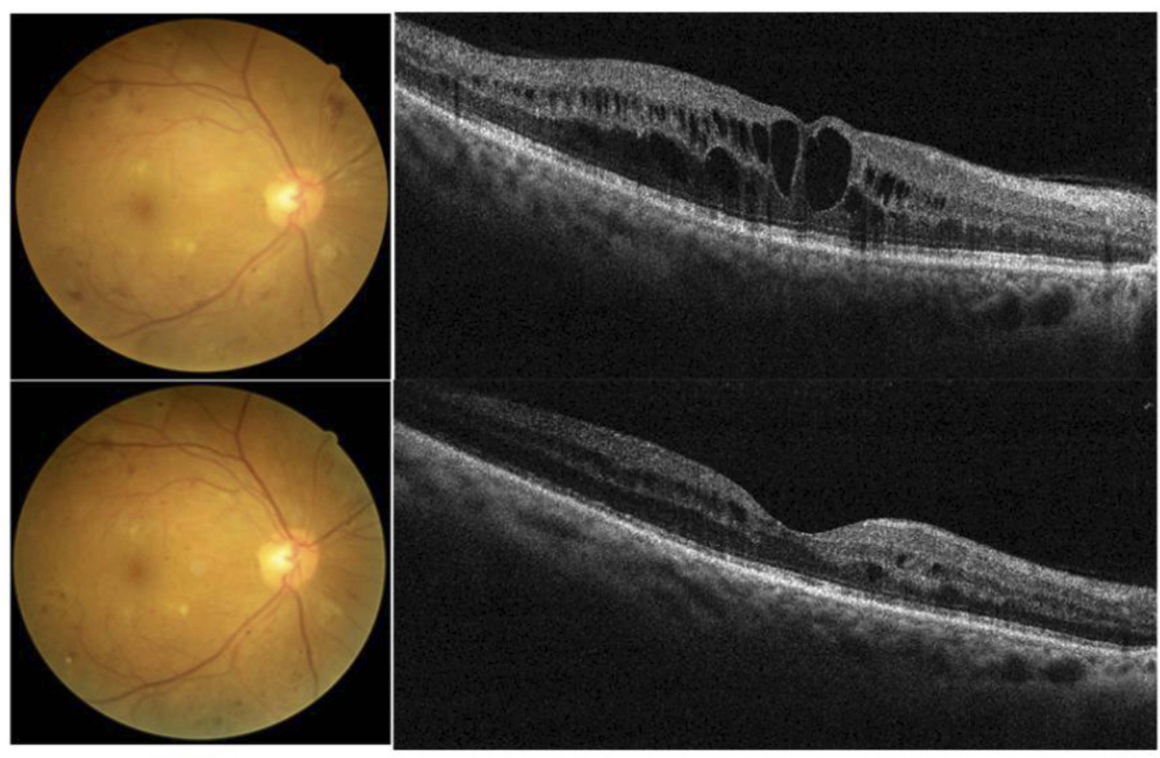

\begin{tabular}{|l|c|c|c|}
\hline & BCVA & CMT & VEGF \\
\hline baseline & 0.05 & 630 & 220.30 \\
\hline
\end{tabular}

Figure 4 Patient presenting with DME. Intravitreal injections at baseline and I month. VEGF levels are given in picograms/millilitre.Abbreviations: BCVA, best-corrected visual acuity (logMAR); CMT, central macular thickness (micrometres). 
monocyte recruitment to the vessel wall after vascular injury. ${ }^{26}$ Together, IL-6, IL-8, eotaxin, G-CSF, IP-10 and MCP-1 act as acute mediators of inflammatory changes and play vital roles as immune modulators. VEGF increases vascular permeability and is implicated in neovascularization. VEGF also plays a critical role in DME development. ${ }^{27,28}$ Large clinical studies have demonstrated that anti-VEGF therapy improves DME. ${ }^{29-31}$ The use of antiVEGF agents as a treatment for DME is recommended as a first-line treatment for centre-involving DME. ${ }^{32}$ Our results suggest that inflammatory factors and VEGF play important roles in the pathogenesis of DME, and these results are in accordance with the results of previous studies. ${ }^{17}$

We also found that the baseline levels of IL-6, IL-8, eotaxin and MCP-1 were significantly correlated with each other in DME. There was also a correlation between the baseline levels of aqueous IL-6 and G-CSF in DME patients. A study showed that the baseline levels of aqueous VEGF were related to IL-6 and IL- 8 in DME. ${ }^{33}$ However, we did not observe an association between VEGF and the baseline levels of IL- 6 or IL-8 in aqueous humour in DME in our study. Therefore, the relationship between the levels of VEGF and inflammatory factors in DME needs further study. These findings suggest the vital role of further investigations into the relationships among inflammatory factors, growth factors, VEGF, and cytokines in DME, and these factors may contribute to a better understanding and treatment of this disease.

Currently, the use of anti-VEGF agents as a treatment for DME is increasing worldwide, and these agents are now widely considered to be the gold standard treatment for DME. ${ }^{6}$ There are many anti-VEGF agents, such as bevacizumab, ranibizumab, aflibercept and conbercept. Conbercept is a fusion protein comprising the extracellular domain-2 of VEGFR-1 and the extracellular domain-3 and 4 of VEGFR-2. Conbercept has a high affinity for placental growth factor, which can act as a chemotactic, mitogenic, or vascular permeability factor in endothelial cells. ${ }^{34}$ Clinical studies have demonstrated that conbercept has good efficacy for improving VA and decreases macular thickness in patients with DMR. ${ }^{35}$ In our study, we also found that BCVA slightly improved following IVC compared with baseline, although this difference was not significant, and CMT significantly decreased 1 month after IVC treatment. CMT significantly decreased and BCVA slightly increased after IVC treatment for DME, which may have been due to our small sample size and needs further study.
To our knowledge, this is the first study to show a significant impact of intravitreal conbercept on the levels of various cytokines in DME. In our study, we found that conbercept therapy reduced only VEGF concentrations without significantly changing the levels of other inflammatory cytokines. This effect of conbercept is similar to that of bevacizumab in the treatment of DME. ${ }^{36-39}$ However, ranibizumab treatment reduces not only the levels of VEGF but also the levels of several other inflammatory cytokines. ${ }^{17}$ This finding suggests that although IVC treatment for patients with DME has considerable benefits, conbercept seems to have limited effects on inflammatory mediators. Therefore, a multiple treatment approach seems reasonable to increase the therapeutic effectiveness.

An additional finding from this study was that the decrease in VEGF levels was associated with a decrease in CMT one month after the first IVC treatment in DME patients. However, there were no significant correlations between the decrease in VEGF levels and the improvement in BCVA one month after the first IVC treatment. There were also no significant correlations between the decrease in CMT and the improvement in BCVA one month after the first IVC treatment. The evidence of a direct correlation between CMT and VEGF levels in response to IVC therapy highlights the value of the OCT parameter for guiding VEGF inhibitory therapy and retreatment regimens.

This study had several limitations. First, aqueous humour is currently the most commonly used intraocular specimen for the study of retinal diseases, but vitreous fluid, as an index of primarily posterior segment pathology, should be considered the ideal sample in such cases. ${ }^{14,40,41}$ However, aqueous sampling is less invasive than vitreous fluid sampling. Therefore, aqueous humour is considered the most appropriate biological sample for examining the intraocular biomarkers of DME. ${ }^{42,43}$ Furthermore, the number of samples was relatively small in our study, and significant differences in cytokine levels may have been undetectable. Finally, the present study was a retrospective comparative study, and the significant results observed here need to be verified in further prospective studies. However, our study on the use of conbercept aimed to provide initial evidence on which cytokines and chemokines are detectable, which seems to be of particular interest for further research.

\section{Conclusions}

In conclusion, we showed differences in various cytokines in the aqueous humour of DME patients compared with that of control patients. Furthermore, our study, for the first 
time, indicates that IVC reduces the levels of VEGF without significantly changing the levels of other inflammatory cytokines or growth factors. The decrease in VEGF levels was associated with a decrease in CMT after the first IVC treatment in DME patients. These findings may contribute to a better understanding of the mechanism of DME and to the development of new treatments.

\section{Acknowledgements}

This work was financially supported by the National Natural Science Foundation of China (No. 81470025), Shanghai Municipal Planning Commission of Science and Research Fund (No. ZY(2018-2020)-ZWB-1001-CF) and Bayannaoer Science and Technology Bureau (No. BKZ2017). The authors also thank AJE for English editing.

\section{Disclosure}

The authors report no conflicts of interest in this work.

\section{References}

1. Klein R, Klein BE, Moss SE. Visual impairment in diabetes. Ophthalmology. 1984;91(1):1-9. doi:10.1016/S0161-6420(84)34337-8

2. Klein R, Klein BE, Moss SE, Cruickshanks KJ. The wisconsin epidemiologic study of diabetic retinopathy. XV. The long-term incidence of macular edema. Ophthalmology. 1995;102(1):7-16. doi:10.1016/ S0161-6420(95)31052-4

3. Bandello F, Cunha-Vaz J, Chong NV, et al. New approaches for the treatment of diabetic macular oedema: recommendations by an expert panel. Eye (Lond). 2012;26(4):485-493. doi:10.1038/eye.2011.337

4. Do DV, Schmidt-Erfurth U, Gonzalez VH, et al. The DA VINCI Study: Phase 2 primary results of VEGF Trap-Eye in patients with diabetic macular edema. Ophthalmology. 2011;118(9):1819-1826. doi:10.1016/j.ophtha.2011.02.018

5. Schmidt-Erfurth U, Lang GE, Holz FG, et al. Three-year outcomes of individualized ranibizumab treatment in patients with diabetic macular edema: the RESTORE extension study. Ophthalmology. 2014;121 (5):1045-1053. doi:10.1016/j.ophtha.2013.11.041

6. Wells JA, Glassman AR, Ayala AR, et al. Aflibercept, bevacizumab, or ranibizumab for diabetic macular edema: two-year results from a comparative effectiveness randomized clinical trial. Ophthalmology. 2016;123(6):1351-1359. doi:10.1016/j.ophtha.2016.02.022

7. Babiuch AS, Han M, Conti FF, Wai K, Silva FQ, Singh RP. Association of disorganization of retinal inner layers with visual acuity response to anti-vascular endothelial growth factor therapy for macular edema secondary to retinal vein occlusion. JAMA Ophthalmol. 2019;137 (1):38-46. doi:10.1001/jamaophthalmol.2018.4484

8. Zhang M, Zhang J, Yan M, et al. A Phase 1 study of KH902, a vascular endothelial growth factor receptor decoy, for exudative age-related macular degeneration. Ophthalmology. 2011;118 (4):672-678. doi:10.1016/j.ophtha.2010.08.008

9. Do DV, Nguyen QD, Boyer D, et al. One-year outcomes of the da Vinci Study of VEGF Trap-Eye in eyes with diabetic macular edema. Ophthalmology. 2012;119(8):1658-1665. doi:10.1016/j. ophtha.2012.02.010

10. Bressler SB, Qin H, Beck RW, et al. Factors associated with changes in visual acuity and central subfield thickness at 1 year after treatment for diabetic macular edema with ranibizumab. Arch Ophthalmol. 2012;130(9):1153-1161. doi:10.1001/archophthalmol.2012.1107
11. Anderson DH, Mullins RF, Hageman GS, Johnson LV. A role for local inflammation in the formation of drusen in the aging eye. $\mathrm{Am}$ $J$ Ophthalmol. 2002;134(3):411-431. doi:10.1016/S0002-9394(02) 01624-0

12. Clermont AC, Cahill M, Salti H, et al. Hepatocyte growth factor induces retinal vascular permeability via MAP-kinase and PI-3 kinase without altering retinal hemodynamics. Invest Ophthalmol Vis Sci. 2006;47(6):2701-2708. doi:10.1167/iovs.05-0071

13. Klaassen I, Van Noorden CJ, Schlingemann RO. Molecular basis of the inner blood-retinal barrier and its breakdown in diabetic macular edema and other pathological conditions. Prog Retin Eye Res. 2013;34:19-48. doi:10.1016/j.preteyeres.2013.02.001

14. Vujosevic S, Simo R. Local and systemic inflammatory biomarkers of diabetic retinopathy: an integrative approach. Invest Ophthalmol Vis Sci. 2017;58(6):BIO68-BIO75. doi:10.1167/iovs.17-21769

15. Joussen AM, Poulaki V, Le ML, et al. A central role for inflammation in the pathogenesis of diabetic retinopathy. FASEB J. 2004;18 (12):1450-1452. doi:10.1096/fj.03-1476fje

16. Jonas JB, Jonas RA, Neumaier M, Findeisen P. Cytokine concentration in aqueous humor of eyes with diabetic macular edema. Retina. 2012;32(10):2150-2157. doi:10.1097/IAE.0b013e3182576d07

17. Lim SW, Bandala-Sanchez E, Kolic M, et al. The influence of intravitreal ranibizumab on inflammation-associated cytokine concentrations in eyes with diabetic macular edema. Invest Ophthalmol Vis Sci. 2018;59(13):5382-5390. doi:10.1167/iovs.17-23325

18. Shimura M, Yasuda K, Motohashi R, Kotake O, Noma H. Aqueous cytokine and growth factor levels indicate response to ranibizumab for diabetic macular oedema. $B r \quad J$ Ophthalmol. 2017;101 (11):1518-1523. doi:10.1136/bjophthalmol-2016-309953

19. Figueras-Roca M, Sala-Puigdollers A, Zarranz-Ventura J, et al. Anatomic response to intravitreal dexamethasone implant and baseline aqueous humor cytokine levels in diabetic macular edema. Invest Ophthalmol Vis Sci. 2019;60(5):1336-1343. doi:10.1167/iovs.18-26215

20. Lee WJ, Kang MH, Seong M, Cho HY. Comparison of aqueous concentrations of angiogenic and inflammatory cytokines in diabetic macular oedema and macular oedema due to branch retinal vein occlusion. Br J Ophthalmol. 2012;96(11):1426-1430. doi:10.1136/ bjophthalmol-2012-301913

21. Remick DG. Interleukin-8. Crit Care Med. 2005;33(12 Suppl):S466S467. doi:10.1097/01.CCM.0000186783.34908.18

22. Baggiolini M. Chemokines in pathology and medicine. J Intern Med. 2001;250(2):91-104. doi:10.1046/j.1365-2796.2001.00867.x

23. Becher B, Tugues S, Greter M. GM-CSF: from growth factor to central mediator of tissue inflammation. Immunity. 2016;45 (5):963-973. doi:10.1016/j.immuni.2016.10.026

24. Gangur V, Simons FE, Hayglass KT. Human IP-10 selectively promotes dominance of polyclonally activated and environmental antigen-driven IFN-gamma over IL-4 responses. FASEB J. 1998;12 (9):705-713. doi:10.1096/fasebj.12.9.705

25. Azzurri A, Sow OY, Amedei A, et al. IFN-gamma-inducible protein 10 and pentraxin 3 plasma levels are tools for monitoring inflammation and disease activity in Mycobacterium tuberculosis infection. Microbes Infect. 2005;7(1):1-8. doi:10.1016/j.micinf.2004.09.004

26. Schober A, Zernecke A. Chemokines in vascular remodeling. Thromb Haemost. 2007;97(5):730-737. doi:10.1160/TH07-02-0085

27. Mitchell P, Bandello F, Schmidt-Erfurth U, et al. The RESTORE study: ranibizumab monotherapy or combined with laser versus laser monotherapy for diabetic macular edema. Ophthalmology. 2011;118(4):615-625. doi:10.1016/j.ophtha.2011.01.031

28. Nguyen QD, Brown DM, Marcus DM, et al. Ranibizumab for diabetic macular edema: results from 2 Phase III randomized trials: RISE and RIDE. Ophthalmology. 2012;119(4):789-801. doi:10.1016/j.ophtha. 2011.12.039

29. Korobelnik JF, Do DV, Schmidt-Erfurth U, et al. Intravitreal aflibercept for diabetic macular edema. Ophthalmology. 2014;121(11):2247-2254. doi:10.1016/j.ophtha.2014.05.006 
30. Diabetic Retinopathy Clinical Research N, Elman MJ, Aiello LP, et al. Randomized trial evaluating ranibizumab plus prompt or deferred laser or triamcinolone plus prompt laser for diabetic macular edema. Ophthalmology. 2010;117(6):1064-1077 e1035. doi:10.1016/ j.ophtha.2010.02.031

31. Ishibashi T, Li X, Koh A, et al. The REVEAL study: ranibizumab monotherapy or combined with laser versus laser monotherapy in Asian patients with diabetic macular edema. Ophthalmology. 2015;122(7):1402-1415. doi:10.1016/j.ophtha.2015.02.006

32. Mitchell P, Wong TY. Diabetic macular edema treatment guideline working G. Management paradigms for diabetic macular edema. Am J Ophthalmol. 2014;157(3):505-513e501-508. doi:10.1016/j. ajo.2013.11.012

33. Noma H, Mimura T, Yasuda K, Motohashi R, Kotake O, Shimura M. Aqueous humor levels of soluble vascular endothelial growth factor receptor and inflammatory factors in diabetic macular edema. Ophthalmologica. 2017;238(1-2):81-88. doi:10.1159/000475603

34. Sun Z, Zhou H, Lin B, et al. Efficacy and safety of intravitreal conbercept injections in macular edema secondary to retinal vein occlusion. Retina. 2017;37(9):1723-1730. doi:10.1097/IAE.00000 00000001404

35. Li F, Zhang L, Wang Y, et al. One-year outcome of conbercept therapy for diabetic macular edema. Curr Eye Res. 2018;43 (2):218-223. doi:10.1080/02713683.2017.1379542

36. Roh MI, Kim HS, Song JH, Lim JB, Kwon OW. Effect of intravitreal bevacizumab injection on aqueous humor cytokine levels in clinically significant macular edema. Ophthalmology. 2009;116(1):80-86. doi:10.1016/j.ophtha.2008.09.036
37. Funk M, Schmidinger G, Maar N, et al. Angiogenic and inflammatory markers in the intraocular fluid of eyes with diabetic macular edema and influence of therapy with bevacizumab. Retina. 2010;30 (9):1412-1419. doi:10.1097/IAE.0b013e3181e095c0

38. Sohn HJ, Han DH, Kim IT, et al. Changes in aqueous concentrations of various cytokines after intravitreal triamcinolone versus bevacizumab for diabetic macular edema. Am J Ophthalmol. 2011;152 (4):686-694. doi:10.1016/j.ajo.2011.03.033

39. Wen J, Jiang Y, Zheng X, Zhou Y. Six-month changes in cytokine levels after intravitreal bevacizumab injection for diabetic macular oedema and macular oedema due to central retinal vein occlusion. Br J Ophthalmol. 2015;99(10):1334-1340. doi:10.1136/bjophthalmol-2014-306341

40. Agrawal R, Balne PK, Wei X, et al. Cytokine profiling in patients with exudative age-related macular degeneration and polypoidal choroidal vasculopathy. Invest Ophthalmol Vis Sci. 2019;60 (1):376-382. doi:10.1167/iovs.18-24387

41. Ghodasra DH, Fante R, Gardner TW, et al. Safety and feasibility of quantitative multiplexed cytokine analysis from office-based vitreous aspiration. Invest Ophthalmol Vis Sci. 2016;57(7):3017-3023. doi:10.1167/iovs.15-18721

42. Campochiaro PA, Hafiz G, Mir TA, et al. Pro-permeability factors in diabetic macular edema; the diabetic macular edema treated with ozurdex trial. Am J Ophthalmol. 2016;168:13-23. doi:10.1016/j. ajo.2016.04.017

43. Hillier RJ, Ojaimi E, Wong DT, et al. aqueous humor cytokine levels and anatomic response to intravitreal ranibizumab in diabetic macular edema. JAMA Ophthalmol. 2018;136(4):382-388. doi:10.1001/ jamaophthalmol.2018.0179

\section{Publish your work in this journal}

Drug Design, Development and Therapy is an international, peerreviewed open-access journal that spans the spectrum of drug design and development through to clinical applications. Clinical outcomes, patient safety, and programs for the development and effective, safe, and sustained use of medicines are a feature of the journal, which has also been accepted for indexing on PubMed Central. The manuscript management system is completely online and includes a very quick and fair peer-review system, which is all easy to use. Visit http://www. dovepress.com/testimonials.php to read real quotes from published authors. 